\title{
Une facette méconnue du travail des traducteurs
}

\section{Sophie Léchauguette}

\author{
Université de Bordeaux 3 \\ France
}

Résumé : Les stratégies éditoriales influencent le processus traductif. L'édition fait travailler des traducteurs littéraires et d'autres qui pratiquent une spécialité méconnue, résistant à tout qualificatif. Il s'agit ici de la présenter et de plaider pour l'organisation de formations préparant les traducteurs à la traduction intersémiotique pour les rendre opérationnels dans les secteurs non littéraires. En effet, les maquettes apportent des contraintes supplémentaires à l'acte de traduction. Les satisfaire demande de repenser le rôle du traducteur. Cet article propose donc de faire évoluer l'image du métier auprès du grand public et des étudiants pour revaloriser l'activité du traducteur d'édition.

Mots- clés : statut professionnel, formation, maquette, illustration, unité de traduction, traduction intersémiotique, socialisation.

\begin{abstract}
Some knowledge of the publishers' strategy is useful for understanding the translation process. Besides literary translators, the publishing industry uses translators whose speciality is hardly known. There is therefore no specific training in this area, yet. This paper presents their work and suggests the introduction of courses in intersemiotic translation. Such courses would help translators specialising in the non literary sector of the publishing industry to deal with the layout of the book, insofar as it bears on the translation process. This paper also hopes to convince the layman to revisit the image of this profession and thus to better appreciate the work of all translators.
\end{abstract}

Keywords: professional status, training, book layout, translation unit, intersemiotic translation, social behaviour.

\section{La société et les traducteurs}

$\mathrm{Si}$, par société, on entend, la majorité des lecteurs, force est de reconnaître qu'ils regardent à peine le nom des traducteurs et l'oublient, sitôt lu. Seul le nom de l'auteur en langue originale reste dans les mémoires. Paradoxalement, nourrie d'innombrables métaphores réunies dans La Traduction en citations (Delisle 2007), l'image du métier se cristallise autour de la figure fantasmée du 
traducteur littéraire. Il jouit d'un prestige social suffisant pour attirer des jeunes gens conscients de la grande difficulté d'un métier exigeant un niveau d'étude correspondant à celui d'ingénieur mais offrant des rémunérations bien inférieures. Le statut juridique du traducteur littéraire, assimiléà un auteur et en tant que tel, payé en droits d'auteur dans le secteur de l'édition, consolide cette représentation valorisante.

Toutefois, l'existence de ce statut unique pour l'édition masque l'existence de deux carrières parallèles. L'attention légitime accordée à la traduction littéraire occulte les autres secteurs. La plupart des traducteurs dits « littéraires », du fait de leur mode de rémunération, ne traduisent pas de littérature. Leur tâche, différente et tout aussi complexe, est méconnue. Cet article se propose de la présenter car elle élargit la notion de traduction. Plus encore que l'activité du traducteur littéraire, son produit, les livres qui en sont issus reflètent notre époque, celle de la mondialisation qui tend à homogénéiser les produits de consommation pour les diffuser à la plus grande échelle possible et maximiser les profits. Il ne s'agit pas ici de faire un mauvais procès aux traducteurs en leur reprochant le contexte dans lequel ils exercent leur activité mais de s'intéresser à celle-ci dans toute sa complexité. La présentation standardisée des ouvrages les amène à pratiquer une forme de traduction un peu particulière puisqu'elle exige la prise en compte de messages textuels et visuels. C'est la traduction intersémiotique.

Ceux qui la pratiquent fournissent la majorité de la production éditoriale et, en dehors de ce secteur, l'« écrasante majorité des textes que les futurs traducteurs auront à traduire une fois sur le marché du travail » (Deslisle 1984, 33). Comme le reste de la société, les aspirants traducteurs, encore extérieurs à la profession, ignorent ce débouché et réagissent avec peu d'enthousiasme quand on le leur présente. En début de formation, des préjugés les conduisent à percevoir la seule traduction littéraire comme une activité noble et attrayante. Le reste, hâtivement qualifié de technique, paraît rebutant. Ce rejet, peut-être hérité de la pensée dominante à la fin du XIX ${ }^{\mathrm{e}}$ siècle exprimée par Schleiermacher, reflète l'attitude souvent condescendante que perçoivent les traducteurs spécialisés dans ce type de traduction de la part de collègues vraiment « littéraires ».

Le mépris qui entoure les traducteurs « littéraires » traduisant autre chose que de la littérature tient à l'amalgame entre le statut du texte en traduction et la difficulté de l'entreprise. On s'imagine souvent que la traduction des œuvres est plus ardue que celles des autres textes. La notion d'art appelant celle de talent, le traducteur littéraire aurait quelque chose de plus que les autres, d'où son prestige. Il ne s'agit pas de diminuer le mérite des traducteurs littéraires qui recréent et font vivre dans leur langue des œuvres étrangères. Bien au contraire, il faut saluer leur créativité. Mais est-il nécessaire de le faire au détriment des autres ? Un moyen de redorer le blason de ces traducteurs perçus 
comme « de seconde zone » serait peut-être de commencer par mettre en évidence les spécificités de leur métier et ses difficultés.

\section{Stratégies éditoriales et processus traductif}

Nous allons donc adopter une approche très pragmatique en examinant les conséquences de la stratégie éditoriale consistant à signer des contrats de co-édition sur le travail de traduction. Ces contrats prévoient la sortie simultanée d'ouvrages identiques traduits en plusieurs langues. Cette stratégie commerciale ajoute des contraintes spécifiques au processus traductif. La première est temporelle. Les délais de traduction sont souvent très courts : il faut donc traduire vite. Cet impératif conduit souvent à répartir le travail entre plusieurs traducteurs, ce qui introduit des contraintes d'harmonisation entre les différentes parties. Les traducteurs sont donc soumis à l'autorité du secrétaire d'édition qui tranche en cas de solutions concurrentes. La seconde est spatiale puisqu'en dehors de la couverture qui change, le livre est rigoureusement le même sur tous les marchés où il sort.

Les traducteurs qui collaborent à ces ouvrages souffrent d'exercer dans un entre-deux qui n'est pas celui de l'entre-deux langues ni de l'entre-deux cultures. Ils sont aussi pénalisés par l'absence d'offre de formation spécifique qui s'explique par le fait que cette spécialité se situe entre deux filières universitaires, avant d'être entre deux carrières. La majorité collabore à des collections dont les thématiques peuvent se rapprocher de celles abordées par des traducteurs en libéral, travaillant sur des documents techniques ou pragmatiques. De ce fait, les étudiants issus des formations LEA seraient peut-être mieux préparés que leurs collègues des filières littéraires, mais, contrairement à eux, ils cherchent plutôt une insertion professionnelle dans d'autres secteurs que l'édition. La séparation des formations entre les départements universitaires ne favorise pas la recherche didactique sur une spécialité hybride qui demande une réflexion pluridisciplinaire.

\section{Invisibles, ignorés, innommés}

Par la suite, dans la vie professionnelle, ces traducteurs doivent se contenter d'un statut d'auteurs au rabais. Leur nom figure rarement sur la couverture avec celui de l'auteur. Ils sont relégués dans l'ours, au côté des intervenants de la chaîne éditoriale. Tâcherons ignorés de tous, ils ont l'impression d'être traités avec condescendance, sinon mépris. Etre parfois mieux payés ne le leur fait pas oublier. Les chercheurs se sont penchés sur leur travail à travers le prisme du type de texte traduit, mais sans les faire davantage exister. On parle donc maintenant de traduction de textes pragmatiques. Pour les traductologues, suivant Delisle, il s'agit de la traduction de textes informatifs valant d'abord par leur contenu informationnel. Leurs auteurs et à fortiori le style de ceux- 
ci ne constituent pas une variable significative. Toutefois, ce terme suscite l'interrogation du profane qui ramène l'inconnu au connu et « traduit » pragmatique par « technique ». Son usage contrarie les linguistes désireux de le réserver à une branche de la linguistique. Pourtant, de nombreux ouvrages publiés dans les collections pratiques relèvent sans conteste de la catégorie pragmatique. D'autres toutefois ne sont ni techniques, ni pragmatiques, sans pour autant être littéraires, mot souvent abusivement limité à la fiction. La qualité de l'écriture des essais, variable selon les auteurs, mérite parfois le qualificatif de littéraire. Ce sont d'ailleurs, à en juger par la liste des lauréats et des titres primés depuis 1981, les seuls ouvrages autres que de fiction, susceptibles de valoir le prix Pierre-François Caillé ${ }^{1}$ à leurs traducteurs.

Les statuts régissant et compartimentant le métier aujourd'hui en France esquissent plusieurs manières de vivre une vie professionnelle de traducteur. Il faut cependant se garder de croire que ces statuts sont mutuellement exclusifs. De nombreux traducteurs d'édition travaillent le littéraire et le non-littéraire. D'autres encore cumulent le statut libéral et celui d'auteur. Certains peuvent alterner statut de salarié et d'indépendant ou devenir fonctionnaires internationaux. La nécessité scientifique de définir un objet d'étude précis induit un cloisonnement qui ne reflète pas la réalité du vécu des professionnels.

La diversité des statuts se confond partiellement avec des spécialisations variées, mais en nombre restreint ${ }^{2}$. Clairement identifiées et associées à des langues de spécialité, on les retrouve dans les intitulés de cours et de formation en traduction. Seule la spécialisation du traducteur d'édition collaborant aux collections non littéraires semble échapper à cette logique puisque les sujets possibles apparaissent illimités. Ainsi, d'un point de vue thématique, la polyvalence est de rigueur. Savoir se former en autodidacte à chaque nouveau contrat, pour maîtriser un lexique souvent inconnu, autant dans la langue de départ que dans la langue d'arrivée, fait partie de ses compétences. Ce traducteur doit aussi être capable de s'adapter à des styles rédactionnels différents pour répondre à l'exigence du cahier des charges des collections auxquelles il collabore. Sa véritable spécialisation semble davantage liée à son aptitude à intégrer de nombreux paramètres relevant de contraintes culturelles,

\footnotetext{
${ }^{1}$ Créée en 1981, la liste des traducteurs et textes récompensés montre qu'ici encore, dans le non-littéraire, seuls les essais peuvent prétendre à quelque reconnaissance http:// www.sft.fr/clients/sft/telechargements/file_front/33769_Laureats_du_Prix_Pi erreFrancois_Caille.pdf.pdf

2 « Les documents qu'ils traduisent traitent de questions très diverses dans les domaines technique, politique, scientifique, social, économique et juridique. » Extrait de la description du poste de traducteur sur le site de l'ONU https:// careers.un.org/lbw/ home.aspx? viewtype=LCEFD\&FId=7. On pourrait ajouter la traduction médicale à cette liste de spécialisations se confondant à un secteur d’activité.
} 
professionnelles et sociales, c'est-à-dire du hors-texte à sa réflexion sur le texte. Un moyen de les identifier est d'examiner l'impact de la publication d'un livre dans une collection, puisque celle-ci constitue un ensemble porteur d'une identité visuelle et thématique qui va influer sur l'écriture.

\section{Traduire pour des collections}

Rarement évoquées dans la littérature traductologique, les collections sont pourtant une variable incontournable si l'on veut rendre compte de la réalité du métier (Léchauguette 2010). L'éditeur leur donne une identité visuelle. Les ouvrages sont identifiables à leur couverture, à leurs dimensions ou encore au papier et aux polices de caractère choisies. L'écriture - des auteurs comme des traducteurs n'échappe pas à cette volonté d'homogénéisation. La nécessité d'harmoniser et de coordonner le travail de plusieurs collaborateurs donne lieu à la production de consignes écrites qui offrent un péritexte riche d'enseignements pour le chercheur. Contrairement aux idées reçues, dans cette niche professionnelle, le traducteur ne fournit pas un effort solitaire. Il doit en référer au donneur d'ordre ou se conformer à la charte de la collection pour trancher entre plusieurs traductions également acceptables.

L'utilisation d'un certain lexique, l'adoption d'un ton, des choix grammaticaux participent de la création de l'identité d'une collection. Secrétaires d'édition et correcteurs interviennent sur le tapuscrit remis par le traducteur pour établir le texte définitif lors de la phase dite de « préparation de copie ». Au-delà du travail de correction proprement dit, ils normalisent la rédaction, lissant le style pour le mettre en adéquation avec celui de la collection. Leur action suit les prescriptions destinées à donner une image de marque du produit qu'est le livre autant à travers son aspect matériel qu'à travers sa prose et obéit à des normes culturelles implicites qui ne font donc l'objet d'aucune consigne particulière de la part du donneur d'ordre. Le traducteur n'a pas nécessairement le dernier mot.

Inacceptable en traduction littéraire, cet état de fait est moins choquant si l'on relativise la notion d'auteur. En dehors des essais, beaucoup d'ouvrages sont des textes de commande écrits par des rédacteurs et non par des auteurs. Le travail postérieur à la remise du tapuscrit du traducteur reproduit les étapes de la mise au point du texte de la version originale, due à un scripteur lui-même soumis au cahier des charges de son éditeur. Quand les relations entre donneurs d'ordre et traducteurs sont courtoises, et que les délais le permettent, les remaniements peuvent être discutés par téléphone ou par mail. On peut le déplorer, mais force est de constater que, dans la vie professionnelle, en dehors du domaine littéraire, l'éditeur demande rarement au traducteur de relire la traduction corrigée dans son intégralité et de signer un bon à tirer. Cette pratique est en recul dès lors qu'il s'agit de 
produire une écriture normée correspondant aux attentes des donneurs d'ordre soucieux de répondre à la demande du public.

Le profil sociologique des destinataires des titres d'une collection est plus ou moins connu, même s'il ne s'agit que d'une construction hypothétique. Le descriptif des ouvrages figurant souvent sur les quatrièmes de couverture renseigne sur le lecteur ciblé, indiquant son degré de familiarité avec le sujet abordé, éventuellement son âge : « Voici un ouvrage pour les amoureux du bois qui souhaitent combiner l'art du tournage à l'art de faire plaisir » (Bowen 2002) ;

«Avoir un autre regard sur l'architecture contemporaine et s'approprier de belles idées pour transformer notre univers quotidien, tel est le but de la collection Archi/design/ déco ${ }^{3}$ ». Le prix de l'ouvrage renseigne sur le milieu social et culturel de l'acheteur. Toute la traduction doit être pensée et rédigée en fonction du lectorat. Le texte doit s'adresser à lui et constituer une véritable communication puisque «le rapport à l'original est davantage contingent que fondamental à l'acte de communication » (Gutt 2000, 98 - nous traduisons $)^{4}$.

Il s'ensuit que lorsque les normes culturelles entre les deux cultures en présence varient, le livre traduit doit procéder aux adaptations nécessaires. Le contrat de coédition imposant aux éditeurs acheteurs des droits de traduction de conserver les mêmes maquettes, c'est le texte de la traduction qui va servir de variable d'ajustement pour corriger par exemple des effets inacceptables induits par l'image. Il peut donc présenter des différences notables avec le texte du livre original. La nature du rapport entre les deux pose un problème à de nombreux étudiants car il est très différent de celui unissant une ouvre et sa traduction. En effet, pour « bien-faire » dans ce nouveau contexte, il faut souvent réécrire et non se contenter de traduire. Une manière d'initier les novices à accepter ce changement de perspective consiste à leur faire utiliser (et non traduire) ce type d'ouvrage. Ils découvrent ainsi les critères de qualité pertinents pour évaluer leur traduction.

\section{Vers une unité de traduction intersémiotique}

La théorie fonctionnaliste est née dans les années 1970 de la volonté d'améliorer la critique des traductions (Reiss, La critique des traductions, ses possibilités et ses limites : catégories et critères pour une évaluation pertinente des traductions). Il s'agissait de fournir des critiques plus justes en adaptant les critères aux différents usages des textes traduits et de leur traduction. Indirectement, en faisant intervenir la notion de types de textes traduits, la traductologie ouvre la porte à la mise en place d'une formation spécialisée répondant aux besoins du secteur non littéraire de l'édition. Il n’a pas échappé aux

3 Texte de présentation de la collection « Archi/design/ déco » des Éditions Place des Victoires, Paris.

4 En original: «the relationship to the original is incidental rather than crucial to the communication act » (Gutt 2000, 98). 
chercheurs que les ouvrages pratiques comportent du texte et de l'image et que la compréhension du lecteur naît de son appréhension simultanée des messages textuels et visuels. Dès 1995, Katharina Reiss évoque le caractère mixte du « texte » et la dimension intersémiotique de la traduction :

Pour les textes écrits qui ne constituent une offre d'information complète que combinés avec des images ou avec des représentations graphiques (livres d'images, bandes dessinées, légendes de diapositives, par exemple) ou avec de la musique (chansons, oeuvres lyriques, etc.), il faut savoir que le texte a été mis en forme d'une manière telle que les divers systèmes sémiotiques sont interdépendants. Or, négliger ces interdépendances, c'est se condamner à produire des traductions inadéquates. $(1995,116)$

On retrouve ce point de vue, initialement exprimé dans la traductologie allemande, chez un chercheur hispanophone :

La réalité textuelle présente dans les diverses langues nous montre que les textes ne sont pas formés exclusivement de signes verbaux, c'est-àdire de mots, mais, souvent et selon le type de texte, ils sont accompagnés de signes non verbaux (images, illustrations, tables, graphiques, etc.). C'est la raison pour laquelle la sémiotique doit aussi jouer un rôle fondamental dans ce que nous pourrions appeler un second niveau d'interdisciplinarité dans la description et l'explication de l'activité traduisante ${ }^{5}$. (Sergio Bolaños-Cuéllar 2004, §8) (nous traduisons)

Un an plus tard, Teresa Tomaszkiewicz conclut « La traduction intersémiotique fait-elle partie de la traductologie ? », article qui constitue un plaidoyer pour l'intégration de la sémiotique dans la traductologie par ces mots : « Il est donc largement temps de revenir sur l'idée de J akobson et d'intégrer dans la recherche traductologique la traduction intersémiotique, jusqu'à présent ignorée ou sous-estimée, tant au niveau pratique que théorique. » $(2005,167)$

C'est parce que notre expérience du métier de traducteur spécialisé dans les secteurs non littéraires de l'édition reflète ces approches théoriques qu'il nous semble utile de répondre à cet appel et de tenter de pallier cette lacune. Un moyen d'y parvenir en joignant la théorie à la pratique est de conceptualiser l'activité traduisante autour du livre et non du seul texte. Il suffit pour cela de revenir sur ce qui constitue l'unité de traduction qui, on le sait, ne se confond pas avec un segment du texte à traduire. Le découpage d'un texte peut avoir une

5 « La realidad textual presente en las diversas lenguas nos indica que los textos no están formados únicamente por signos verbales, es decir, por palabras, sino que, a menudo y dependiendo del tipo de texto, están acompañados de signos no verbales (imágenes, ilustraciones, tablas, gráficas, etc.), por lo cual la semiótica también está llamada a desempeñar un papel crucial en lo que podríamos denominar un segundo nivel de interdisciplinariedad en la descripción y explicación del proceso traductor. » 
utilité pédagogique ou permettre une évaluation de la compétence en langue des étudiants mais ne contribue guère à la réflexion traductologique. L'unité de traduction est une entité abstraite qui se construit au fur et à mesure de la découverte d'un texte à traduire et du processus traductif. Des récurrences à plusieurs pages de distance peuvent constituer une unité de traduction puisqu'il faudra reproduire l'effet de récurrence dans la traduction. Ainsi, l'unité de traduction fait le lien entre texte de départ et texte d'arrivée. Résultant de rapports établis par le traducteur, elle intègre sa subjectivité:

L'unité de traduction est un élément constituant d'un tout qui a sa source, ou base formelle, dans le texte de départ, son aboutissement dans le texte d'arrivée, et qui passe pour sa réalisation par le cerveau du traducteur : il s'agit donc d'un ensemble à configuration variable selon l'individu qui le construit; ce qui signifie qu'il faut intégrer la subjectivité dans l'UT. [...] Cela signifie que, sur le plan formel, il existe plusieurs types d'UT selon que leur base apparente est dans le texte de départ (et c'est le plus souvent le cas) ou plutôt générée par la constitution du texte d'arrivée et les exigences extralinguistiques de la culture d'accueil. (Ballard 2006, 125)

Pour le traducteur d'édition, l'unité de traduction se construit à partir des contraintes de rédaction du texte d'arrivée qui doit se couler dans une maquette qui n'est pas conçue pour lui. Elle intègre aussi les exigences extralinguistiques des donneurs d'ordre et de la culture d'accueil. En insistant sur la double appartenance de l'unité de traduction et sur sa constitution par l'activité traduisante, cette définition est assez accueillante pour intégrer une dimension sémiotique. Elle reconnaît l'existence de plusieurs sortes d'unité de traduction et s'appuie sur l'interprétation des formes. Rien n'interdit d'y faire entrer des formes non linguistiques, comme le bloc ou pavé de texte, dès lors qu'elles contribuent à la construction du sens. Il est donc possible d'intégrer l'environnement spatial du texte que constitue la double-page, unité de base d'une maquette à la réflexion traductologique. Dans la mesure où cet environnement - découpage du texte et intégration des blocs à un dispositif illustré - apporte des contraintes supplémentaires au traducteur, il faut le prendre en compte. Avant même d'approfondir l'interaction entre le texte et les illustrations, signalons que l'éclatement du message textuel en rubriques hiérarchisées disposant d'un espace identique pour les éditions en différentes langues induit une contrainte d'encombrement.

\section{Traduire avec la maquette}

Traduire pour un ouvrage maquetté nécessite une compréhension du vocabulaire typographique et de la manière dont le tapuscrit va être traité en aval, par le maquettiste chargé de la fabrication du livre. La première contrainte découlant du format du 
livre est la nécessité de tenir compte de l'encombrement du texte. Le nombre de caractères maximum possible est un multiple du nombre de lignes disponibles. Suivant les rapports de foisonnement entre les langues en présence, le traducteur doit donc pratiquer la traduction résumante, pour que son texte ne chasse pas ou, inversement, peut-être l'incrémenter pour ne pas laisser trop de vide. D'autres contraintes d'ordre typographique peuvent influer sur la rédaction de la traduction. Quand un texte linéaire chasse, l'éditeur peut réduire le corps de caractère afin de résoudre le problème et faire tenir la traduction dans le nombre de pages imparti. Toutefois, si la priorité est d'utiliser un corps de caractères prédéfini, c'est le texte qui devient la variable d'ajustement. Quand il s'étale sur plusieurs pages, le maquettiste joue avec l'espace en utilisant les lignes souvent disponibles en fin de chapitre. Quand le bloc de texte s'inscrit dans des cadres, matérialisés ou non, ou des colonnes, sa marge de manœeuvre est moindre.

Dans les manuels, la présentation de la double-page type se répète du début à la fin du livre ou presque. Elle comporte généralement (en page paire, à gauche) un titre, un chapeau ou soustitre, du texte courant, des légendes. Les guides pratiques ajoutent souvent (en page impaire à droite) une rubrique appelée pas-à-pas présentant les étapes d'une réalisation illustrées de photos. Titres et chapeaux, si l'on reprend la typologie de Reiss, ont une fonction appellative. Il s'agit d'arrêter le lecteur, de lui donner l'envie de réaliser le projet proposé (une recette dans un livre de cuisine). Le texte courant a plutôt une fonction informative. Quand ce texte linéaire coexiste avec un pas-à-pas, il présente le texte découpé qui le reprend, mais sous forme d'instructions.

Ces deux rubriques sont dans un rapport de redondance qui pousse à la répétition, à proscrire quand on rédige en français. Il faut donc trouver des moyens de les différencier. On utilise généralement la troisième personne (parfois à la première) du mode indicatif pour le texte courant et on réserve l'infinitif ou l'impératif aux instructions. Ces décisions échappent aux traducteurs car elles sont prises en amont, et lui sont communiquées de manière formelle ou informelle. Quand des encadrés occupent l'espace des marges, suivant les ouvrages, ce sont des textes anecdotiques venant « illustrer » le propos de la double page ou des rappels et résumés de son contenu. Ils sont là pour enjoliver le livre ou valoriser une information. Les premiers renvoient parfois à des particularités de la culture de départ dénués de pertinence une fois traduit pour un lectorat aux références culturelles différentes. La meilleure traduction est alors une substitution. Le travail du traducteur est de rechercher une information équivalente dans la culture destinataire puis d'écrire un texte qui remplacera le texte initial. Il faut bien sûr restituer le contenu informationnel des seconds, mais peutêtre en le réécrivant pour éviter un effet de répétition, qui ne gêne pas les lecteurs anglophones mais passe très mal auprès des francophones. 


\section{De la traduction au métier de traducteur}

Pour les professionnels, ces interventions sur le texte vont de soi. Elles ressortent de l'adaptation, implicitement couverte par le mot traduction. Il est également considéré comme normal ou du moins inévitable que le livre en traduction contienne des inexactitudes. Qu'on le lui dise ou pas, le traducteur est chargé de les repérer et de corriger, de préférence en les signalant à l'éditeur. Il n'est pas rare de constater des inversions entre les textes des instructions et les images des pas-àpas. Pour les remarquer, il faut traduire en s'appuyant et en confrontant messages textuels et visuels. L'unité de traduction des encyclopédies, guides et manuels pratiques ... publiés en dehors des collections littéraires est donc bien mixte. Inhérente à la fabrication du livre, cette particularité constitue la caractéristique définissante de la traduction pour l'édition sans toutefois se limiter à ce secteur. Cette problématique peut être élargie aux traducteurs libéraux, quand ils traduisent des brochures, et aux traducteurs techniques dont l'activité consiste à traduire des brevets ou des manuels. Ils travaillent davantage avec des schémas, des croquis ou des dessins qu'avec des photos en quadrichromie mais eux aussi doivent penser le texte en fonction des illustrations. Il apparaît donc d'autant plus nécessaire de passer de la pensée théorique à la pratique par la mise en place de formations spécialisées.

\section{Vers un cours de traduction qui intègre la dimension intersémiotique du métier}

Pour des personnes en formation issues de filières littéraires, en voie de professionnalisation, le vécu quotidien du traducteur d'édition est déroutant. Leur passé d'étudiants en langues, bons en version, les conduit à aborder la formation avec les images du métier véhiculées par les métaphores évoquées plus haut. Leurs représentations sont aussi fausses que celles des profanes. Leur réaction quand on leur explique ces réalités vont de la surprise au refus. Au stade initial, la formation doit d'abord réconcilier les images en décalage avec le métier et sa réalité. Il faut convaincre que la tâche des traducteurs d'édition ne se limite pas à la traduction des ouvres littéraires et que les autres collections offrent des débouchés intéressants et lucratifs. Il faut revenir sur le rôle du traducteur et montrer comment son travail s'insère dans la chaîne éditoriale qui aboutit à la mise sur le marché d'un livre. Travaillant chez lui, le traducteur n'a pas la possibilité d'apprendre à gérer les relations professionnelles au contact de ses collègues comme les autres jeunes diplômés. Il est donc nécessaire d'expliquer les rapports entre les différents intervenants sans omettre la question des délais. La vie professionnelle exige une rapidité à laquelle les aspirants traducteurs ne sont pas habitués. Une fois 
acceptés ces préliminaires qui relèvent d'une socialisation seconde (au sens de Dubar), le terrain est prêt pour aborder la traduction des ouvrages dans sa dimension communicationnelle et sémiotique.

Il est temps de passer à l'apprentissage de techniques de traduction et à la transmission de savoir-faire par des exercices pratiques toujours suivis de discussions collectives et de phases de réécriture. Le support idéal de l'exercice est l'ouvrage en entier, ou, à défaut, des reproductions en couleur de l'intégralité des pages d'où sont tirés les passages retenus pour les exercices. Quand chaque étudiant s'entraîne sur des extraits d'ouvrages différents, la révision des travaux d'autres étudiants est un moyen de développer les capacités critiques et les qualités d'écriture des étudiants. En les plaçant en situation de relecteurs/ correcteurs, on les libère de la tyrannie du texte de départ. On leur permet de dépasser leur tendance initiale au transcodage linguistique et de mobiliser leur bon sens parfois mis en échec par la difficulté à s'approprier les informations transmises. Les maladresses initiales dans des premiers travaux rendus, où les étudiants n'ont manifestement pas mis leur texte en relation avec l'illustration, témoignent de la nécessité d'apprendre à traduire avec des images. L'expérience montre que les étudiants appréhendent mieux le travail attendu quand le cours ne se limite pas à des activités de traduction mais comprend aussi des phases d'utilisation des ouvrages, avec réalisations pratiques à l'appui et des phases de travail éditorial (correction, réécriture). L'organisation des activités proposées en atelier autour des rubriques de la maquette aide à mieux faire percevoir leurs différentes visées communicatives. Il est ensuite plus facile d'adopter un style rédactionnel adéquat. Le traducteur d'édition spécialisé dans les secteurs non littéraires ne traduit pas que des ouvrages pragmatiques, mais il doit faire preuve d'un grand pragmatisme. C'est à l'enseignant formateur de l'aider à développer cette compétence rarement répertoriée parmi celles du traducteur.

Peut-être vaudrait-il mieux parler de traducteurs pragmatiques que de traduction pragmatique? Dans ce secteur, la débrouillardise est de mise. Le travail de documentation nécessaire à la compréhension du texte à traduire puis à sa transmission à un nouveau public, culturellement distinct de ses destinataires, demande d'avoir de la ressource. Ce traducteur fait parfois un véritable travail de limier pour identifier un objet... puis trouver comment s'en passer et par quoi le remplacer, quand il n'est pas disponible dans la culture d'arrivée. C'est le cas de bien des ingrédients nécessaires à la réussite des plats exotiques. Il doit faire preuve d'initiative, de sens critique et d'imagination, compétences difficiles à enseigner mais que l'on peut développer en proposant des exercices qui testeront les capacités des apprenants à mobiliser tout leur bagage cognitif.

Les diverses activités proposées visent à inviter les personnes en formation à sans cesse faire le lien entre le livre, c'est-à-dire le texte qu'ils rédigent (ou devront rédiger), les images qui l'entourent et 
l'extra-inguistique : le monde réel dans lequel le lecteur utilisateur tentera de mettre en œuvre les instructions écrites. Le test de la qualité d'une traduction d'un manuel est l'aisance avec lequel ses lecteurs comprennent les instructions. La facilité à les exécuter affirme le succès de la traduction mieux que toute comparaison entre celle-ci et un texte de départ que les interventions pertinentes et motivées des traducteurs auront fait oublier. Pour apprendre à bien rédiger, les traducteurs novices doivent commencer par prendre toute la mesure de leur futur métier.

\section{Traducteurs d'édition : experts en traduction inter- sémiotique}

Autant que la traduction littéraire, la traduction intersémiotique pose constamment de nouveaux défis aux traducteurs. Elle exige initiative et créativité, sens critique et aptitude au travail en équipe. Elle taxe leur capacité cognitive pour comprendre et se documenter avant de restituer les contenus informationnels dans le style convenant au type d'ouvrage en traduction. La traduction intersémiotique demande en outre des compétences techniques liées aux métiers de l'édition que sont la typographie et le travail du maquettiste. Elle place le traducteur en position charnière entre la rédaction du texte et sa composition. Il est grand temps de reconnaître les spécificités de cette spécialité pour faire sortir ceux qui la pratiquent de la double invisibilité où les relègue une ambiguïté juridico-linguistique qui les désigne trop souvent par l'amalgame avec les traducteurs littéraires. Il faudrait peut-être, suivant Ladmiral (2005), parler « d'experts en communication multilingue » et intersémiotique pour qu'enfin soit reconnu le large éventail de leurs compétences et que leur travail bénéficie du respect qu'il mérite. Ces traducteurs bénéficieraient alors d'une reconnaissance sociale suffisante pour susciter des vocations.

\section{Références bibliographiques}

Ballard, Michel. «A propos des procédés de traduction ». In : Christine Raguet, Palimpseste. Traduire ou Vouloir garder un peu de la poussière d'or. Vol. Hors série. Paris : Presses de la Sorbonne Nouvelle, 2006 : 113-30.

Bolaños-Cuéllar, Sergio. « Hacia una visión integradora de la traducción: propuesta del modelo traductológico dinámico (mtd). » [Vers une vision intégratrice de la traduction: proposition pour un modèle traductologique dynamique]. Revista electronica de estudios filologicos (2004). URL : http:// www.revistas.unal.edu.co/index.php/ formayfuncion/ article/ view/ 17 286/ 18125. (Consultéle 29 aout 2013).

Bowen, H. Bijoux en bois tourné. Paris : Eyrolles, 2002, coll. « Collection Idées et Modèles ». 
Delisle, J ean. La traduction en citations. Ottawa : Presses de l'Université d'Ottawa, 2007.

Delisle, J ean. L'Analyse du discours comme méthode de traduction Théorie et Pratique. Ottawa : Edition de l'université d'Ottawa, 1984.

Dubar, Claude. La socialisation. Collection U. 4e. Paris : Armand Colin, 2010. Gutt, Ernst-August. Translation and Relevance Cognition and Context. [Traduction et pertinence. Cognition et contexte]. Manchester : St J erome, 2000.

Ladmiral, J ean-René. «Former des traducteurs : pour qui ? pour quoi ? ». Meta, 2005, 50, 1: 28-35. Montréal : Presses de l'université de Montréal. URL : http:/ / www.erudit.org/ revue/ meta/ 2005/v50/n1/ 010654ar.html, (Consulté le 11 octobre 2013).

Léchauguette, Sophie. « Traduire pour des collections pratiques. 》 Translationes 2 (2010) : 89-104.

Reiss, Katharina. La critique des traductions, ses possibilités et ses limites : catégories et critères pour une évaluation pertinente des traductions. Traduit par Catherine Bocquet. Traductologie. Arras : Artois Presses Universitaires, 2002.

Reiss, Katharina. Problématiques de la traduction. Bibliothèque de traductologie. Préface de J ean-René Ladmiral. Traduit par Catherine Bocquet. Paris : Economica - Anthropos, 1995.

Schleiermacher, Friedrich Daniel Ernst. « Des différentes méthodes du traduire. » (1899). In : Les tours de Babel, essais sur la traduction. Traduit par Antoine Berman. Trans-Europ-repress, Mauvezin. Paris : Seuil, 1985.

Tomaszkiewicz, Teresa. « La traduction intersémiotique fait-elle partie de la traductologie? ». In : J ean Peeters. La traduction de la théorie à la pratique et retour. Rennes : Presses universitaires de Rennes, 2005 : 159-68. 